\title{
Correction to: Cost-Utility and Budget Impact Analysis for Stopping the Inappropriate Use of Proton Pump Inhibitors After Cessation of NSAID or Low-Dose Acetylsalicylic Acid Treatment
}

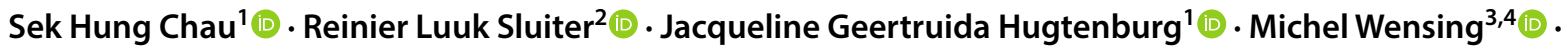 \\ Wietske Kievit ${ }^{2}$ (I) Martina Teichert ${ }^{5}$
}

Published online: 5 November 2019

(c) The Author(s) 2019

\section{Correction to: Drugs \& Aging https://doi.org/10.1007/s40266-019-00713-5}

Errors were subsequently identified in the article originally published online, and the following corrections should be noted:

\section{Correction to author name:}

Page 1, author listing and corresponding author details: The name of the first and corresponding author was incorrectly formatted and should read:

Sek Hung Chau.

\section{Correction to Figure 1:}

Page 3, Fig. 1: An arrowhead was omitted between the "Healthy" and "Hip fracture" health states in the figure. The corrected figure is shown here.

The original article can be found online at https://doi.org/10.1007/ s40266-019-00713-5.

Sek Hung Chau

s.chau@amsterdamumc.nl

1 Department of Clinical Pharmacology and Pharmacy, Amsterdam Public Health Research Institute, Amsterdam UMC, Vrije Universiteit Amsterdam, De Boelelaan 1117, Amsterdam, The Netherlands

2 Department for Health Evidence, Radboud University Medical Center, Geert Grooteplein 21, Nijmegen, The Netherlands

3 Radboud University Medical Center, Scientific Institute for Quality of Healthcare (IQ Healthcare), Geert Grooteplein 21, Nijmegen, The Netherlands

4 Department of General Practice and Health Services Research, Heidelberg University, Im Neuenheimer Feld 130.3, Heidelberg, Germany

5 Department of Clinical Pharmacy and Toxicology, Leiden University Medical Center, Albinusdreef 2, Leiden,

The Netherlands

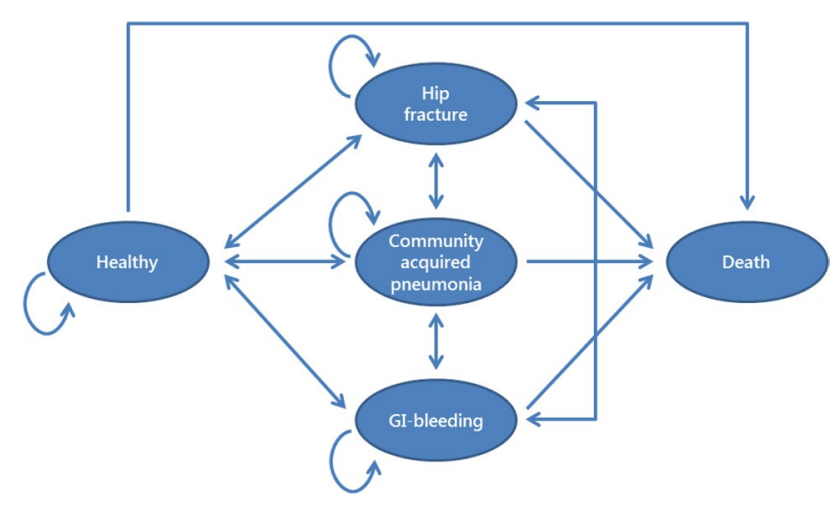

Open Access This article is distributed under the terms of the Creative Commons Attribution-NonCommercial 4.0 International License (http://creativecommons.org/licenses/by-nc/4.0/), which permits any noncommercial use, distribution, and reproduction in any medium, provided you give appropriate credit to the original author(s) and the source, provide a link to the Creative Commons license, and indicate if changes were made. 